\title{
Oral Mucositis: Role of the Dentist
}

\author{
Sathyasree Madeswaran ${ }^{1}$, Deepshika Saravanan ${ }^{2}$, Saravanan Rethinam ${ }^{3}$, Kavitha Muthu ${ }^{4}$
}

\begin{abstract}
Oral mucositis is the inflammation of the oral mucosa that occurs as a side effect of cytotoxic cancer therapy. Though the condition cannot be prevented, steps can be taken to decrease the severity and for the desired therapeutic outcome of cancer therapy. The communication between the oncologist and dentist becomes vital for the patient benefit. A search of the resources through Medline and Google Scholar was made to comprehend the pathobiology, clinical characteristics, and various therapeutic methods related to oral mucositis. A total of 63 articles were chosen for reference, which mostly includes recent year review papers. After the final screening, 29 research papers were selected for the current study. Controlling the predisposing risk factors pertaining to the oral cavity and recording the baseline oral health status before the commencement of cancer therapy is crucial. Patients are counseled for proper oral hygiene routine, and lifelong follow-up.

Keywords: Chemotherapy, Oral health, Oral mucositis, Radiation therapy.

Journal of Oral Health and Community Dentistry (2019): 10.5005/jp-journals-10062-0057
\end{abstract}

\section{INTRODUCTION}

Oral mucositis is a common, reversible, but debilitating side effect that occurs in the oral cavity due to radiation and chemotherapy. Proportionate to the severity of its occurrence, it can significantly contribute to the morbidity of the patients. ${ }^{1}$ Although the target for radiation and chemotherapy is the rapidly multiplying cancer cells, the tissues with rapid division potential like the oral and gastrointestinal mucosa, skin, bone marrow, hair follicles, and the tissues adjacent to the target area are also affected. ${ }^{2}$

Oral mucositis occurs in almost all patients receiving head and neck radiation therapy, $20-40 \%$ of patients undergoing chemotherapy, and in $80 \%$ of patients undergoing the myeloablative chemotherapy. ${ }^{3,4}$ Hematopoietic stem cell transplantation is a treatment procedure done to rebuild the bone marrow for patients suffering from lymphoma, leukemia, and many non malignant disorders. As a routine, before transplantation, patients undergo myeloablative conditioning regimen to eliminate the tumor cells, to create space for the transplantation, and to induce immunosuppression for overcoming host rejection response. ${ }^{5}$ It is done by using chemotherapeutic drugs with or without total body irradiation.

\section{Oral Mucosa and Mucositis}

Oral mucosa lines the oral cavity and serves to protect the deeper tissues from friction and abrasion arising due to mechanical forces. More importantly, it serves as a protective barrier preventing the entry of microorganisms and exogenous molecules into the systemic circulation. ${ }^{6}$ In the oral cavity, the epithelial cells of the nonkeratinized lining mucosa have a less turnover time (14 days) than that of the cells of keratinized masticatory mucosa (24 days). Hence, the effect of cancer therapy on nonkeratinized mucosa is more profound. The highly keratinized hard palate, dorsum of the tongue, and the gingiva are generally spared. ${ }^{6}$ Cell death is caused by damage to DNA, apoptosis, and bystander effect (cells that are not directly irradiated or targeted by chemotherapeutic drugs exhibit altered response in terms of proliferation, gene expression, etc.). ${ }^{2}$ Table 1 describes the difference between chemotherapy- and radiation-induced mucositis. \begin{tabular}{l}
\hline \hline Department of Prosthodontics, Crown and Bridge, RVS Dental College \\
and Hospital, Coimbatore, Tamil Nadu, India \\
${ }^{2}$ Department of Periodontology, RVS Dental College and Hospital, \\
Coimbatore, Tamil Nadu, India
\end{tabular}

${ }^{3}$ Department of Oral Surgery, RVS Dental College and Hospital, Coimbatore, Tamil Nadu, India

${ }^{4}$ Department of Oral Pathology, RVS Dental College and Hospital, Coimbatore, Tamil Nadu, India

Corresponding Author: Kavitha Muthu, Department of Oral Pathology, RVS Dental College and Hospital, Coimbatore, Tamil Nadu, India, Phone: +91 87541 34965, e-mail: Kavidentist@yahoo.co.in

How to cite this article: Madeswaran S, Saravanan D, Rethinam S, et al. Oral Mucositis: Role of the Dentist. J Oral Health Comm Dent 2019;13(3): 106-111.

Source of support: Nil

Conflict of interest: None

\section{Significance}

Severe oral mucositis can alter the course of cancer treatment and its outcome by disrupting the treatment regimen due to local and systemic infections. Ulcer formation and microbial colonization cause systemic infection leading to hospitalization. Also, severe pain can warrant the use of opioid analgesics, preclude oral nutrition necessitating the use of other modes like a parenteral and nasogastric feeding tube. It significantly adds to the cost of treatment. ${ }^{10,11}$

\section{Pathobiology and Clinical Course}

Earlier paradigm was that mucositis occurred due to the direct injury caused by radiation and chemotherapy to the epithelial cells causing clonogenic cell death. The imbalance created by more cell death than replacement results in the loss of continuity of epithelium, leading to ulcer formation. ${ }^{12}$ Infection as an etiologic factor was also proposed and has been disproved. ${ }^{13}$ The currently accepted paradigm in the pathogenesis of oral mucositis proposed by Sonis ${ }^{14}$ is characterized by five phases.

o The Author(s). 2019 Open Access This article is distributed under the terms of the Creative Commons Attribution 4.0 International License (https://creativecommons. org/licenses/by-nc/4.0/), which permits unrestricted use, distribution, and non-commercial reproduction in any medium, provided you give appropriate credit to the original author(s) and the source, provide a link to the Creative Commons license, and indicate if changes were made. The Creative Commons Public Domain Dedication waiver (http://creativecommons.org/publicdomain/zero/1.0/) applies to the data made available in this article, unless otherwise stated. 
Table 1: Difference between chemotherapy- and radiation-induced mucositis

\begin{tabular}{l} 
Chemotherapy-induced mucositis \\
\hline Due to systemic administration of the drug lesions are not \\
site-specific, can occur bilaterally in the oral cavity. Affects \\
nonkeratinized lining mucosa (i.e.) lateral border and ventral \\
surface of the tongue, the floor of the mouth, buccal mucosa and soft \\
palate \\
Acute onset. Arises within $4-5$ days following infusion and \\
ulcerates 5 days later ${ }^{8}$ \\
Incidence is higher and severe with selected agents such as \\
antimetabolites and alkylating agents. Severity is proportional to \\
neutropenia and drug toxicity \\
Subsides gradually in $2-3$ weeks in the absence of bone marrow \\
suppression \\
Heals spontaneously without scar formation
\end{tabular}

\section{Initiation}

Chemotherapy and radiation cause direct DNA damage resulting in the immediate death of cells in basal ganglia and submucosa. More potential damage starts to occur due to the generation of reactive oxygen species (ROS). The ROS damage the DNA, connective tissue, cell membrane, and triggers the macrophages and many inflammatory cascades. Clinically, the mucosa appears normal and quiescent, which is a striking contrast to the multitude of events taking place in the submucosa.

\section{Primary Damage Response}

The ROS triggers the production of proinflammatory molecules and subsequent pathways resulting in the upregulation of inflammatory mediators. Nuclear factor- $\mathrm{KB}$ is one such important biologically active molecule that is produced due to cell damage. It is responsible for the expression of proinflammatory genes and cytokines, such as tumor necrosis factor- $a$, interleukin- 6 , and interleukin-1 $\beta$. The enzymes sphingomyelinase and ceramide synthase are activated to produce ceramide, a lipid molecule present in the cell membrane. The ceramide pathway plays an important role in apoptosis, also termed as programmed cell death. Damage to the fibroblasts results in the secretion of matrix metalloproteinases (MMPs) responsible for the disruption of proteins in the extracellular matrix.

\section{Signal Amplification}

The inflammatory mediators activate other potent molecules and enhance the signaling pathway through positive feedback resulting in additional injury. These feedback loops continue to provide the destructive signal even after the cessation of irradiation and chemotherapeutics. The mucosa appears erythematous and edematous due to changes in capillary permeability.

\section{Ulceration}

The death of endothelial cells lining the blood vessels in the submucosa results in reduced secretion of the keratinocyte growth factor (KGF) leading to reduced regeneration of epithelial cells. Reduced regeneration, clonogenic cell death, and apoptosis result in the degeneration of the epithelium. Destruction of submucosa and epithelial degeneration leads to ulcer formation. The dead cells and fibrin form a pseudomembrane over the ulcers. Bacteria proliferate and activate the macrophages to produce more proinflammatory mediators. This is the most symptomatic phase
Radiation-induced mucositis

Site-specific. Limited to the mucosa in the field of radiation ${ }^{7}$

Insidious onset. Mucosal changes occur at end of the first week and peaks at the end of the third week after attaining a cumulative dose of $30 \mathrm{~Gy}^{8}$

Severity is proportional to the dosage, duration and field of radiation 8

Heals within 2 months ${ }^{2}$

As a late sequel, the mucosa can become atrophic, relatively avascular, fibrous and more prone to develop chronic ulcers ${ }^{2}$

Table 2: WHO (1979) classification of oral mucositis

\begin{tabular}{ll}
\hline Grade & Description \\
\hline O (none) & None \\
I (mild) & Oral soreness, erythema \\
II (moderate) & Oral erythema, ulcers, solid diet \\
& tolerated \\
III (severe) & Oral ulcers, liquid diet only \\
IV (life-threatening) & Oral alimentation impossible \\
\hline
\end{tabular}

characterized by severe pain. It carries the risk of septicemia due to the invasion of bacteria into the submucosal blood vessels.

\section{Healing}

After the completion of cancer therapy, angiogenesis and downregulation of inflammatory signals occur. Growth signals from the connective tissue matrix lead to the proliferation and differentiation of basal cells. The ulcers heal spontaneously, but the mucosa assumes a slightly altered form that makes it more vulnerable for future assault. Table 2 describes the WHO classification of oral mucositis.

\section{Contributing Risk Factors}

Oral mucositis occurs in varying severity depending on the dose and duration of the chemotherapeutic drug and radiation. Many factors attribute to the severity of the condition. Age, xerostomia, neutropenia, pancytopenia, secondary infections, poor baseline nutritional status, mucosal trauma, poor oral hygiene, and periodontal disease contribute to the severity.

\section{Age}

Young patients are at increased risk due to a higher mitotic rate of the epithelium. The lesions also heal faster due to the same reason. On the contrary, older patients undergoing chemotherapy also face higher risk due to decreased cell turnover rate and a general decline in kidney function resulting in increased drug toxicity. ${ }^{15}$

\section{Xerostomia}

Saliva is the key regulator of oral health. Cancer therapy can result in changes in the saliva leading to an increase in microbial load, altered microbial flora, and oral physiology. Radiation destroys the serous 
cells of the salivary glands, as they are more radiosensitive than mucous cells. Hence, the saliva decreases in quantity and becomes more viscous. The buffering capacity of the saliva is reduced. There is a shift in the $\mathrm{pH}$ from an average of $6.5-5.5$. $^{2}$ This can lead to the onset of the demineralization of teeth. The lack of lubrication leads to difficulty in swallowing, pain, and mucosal trauma. Saliva also plays a role in antimicrobial defense. ${ }^{2,15}$ While the condition improves after the completion of chemotherapy, it may not in some cases of radiation therapy. This is because of permanent damage to the salivary gland tissue. ${ }^{16}$

\section{Neutropenia}

Neutropenia is diagnosed if the neutrophil count in the blood is less than $1,500 / \mathrm{mm}^{3}$ and is considered severe if the count is less than $500 / \mathrm{mm}^{3}$. It is a common side effect of chemotherapy. It can also occur if radiation is given in multiple active bone marrow proliferation sites. Febrile neutropenia is a condition in which fever is associated with neutropenia. This is considered an oncology emergency as it indicates systemic infection. Though the condition is not preventable, it can be managed without serious complications by prompt diagnosis, hospitalization, and administration of antibiotics. ${ }^{17}$ Febrile mucositis is a clinical entity in which mucositis presents with fever. The ulcerated mucosa becomes a portal of entry for the microorganisms to enter, the systemic circulation causing septicemia. Neutropenia can exacerbate the condition. ${ }^{18}$

\section{Pancytopenia}

Pancytopenia is decreased in the number of erythrocytes, leukocytes, and platelets. This can occur due to myelosuppressive chemotherapy and total body radiation. ${ }^{19}$ The patient presents with shortness of breath, malaise, fatigue, fever, infection, and a tendency to bleed. The gingiva acquires a tendency to bleed, making even brushing difficult. In the case of severe ulcerative mucositis, bleeding occurs in the oral cavity. Spontaneous bleeding and persistent bleeding lasting more than 2 minutes indicate the need for whole blood or platelet transfusion. ${ }^{20}$

\section{Secondary Infection}

Herpes simplex virus, Candida albicans, and the normal microbial flora proliferate and cause opportunistic infection in immunocompromised patients. These infections can prolong the duration of mucositis and can present additional symptoms like dysgeusia, angular cheilitis, erythema, and ulcers involving the keratinized mucosa, which is usually spared in oral mucositis. ${ }^{1}$

\section{Malnutrition}

Malnutrition, a state of negative energy and nutrient balance, impairs the immune response and increases any treatmentrelated morbidity. ${ }^{21}$ Cancer is a disease characterized by altered metabolism, increased inflammation, and catabolism leading to anorexia and progressive cachexia. The cytotoxicity induced by cancer therapy can further bring down the nutritional status of the patient. ${ }^{22}$

\section{Mucosal Trauma}

The connective tissue of the lining mucosa is flexible and elastic to accommodate the bolus of food for grinding and swallowing. This makes the lining mucosa more prone to traumatic injury when compared to the masticatory mucosa, which is tightly attached to the underlying bone. ${ }^{23}$ Sharp teeth, faulty restorations, ill-fitting dentures, misaligned teeth, coarse and hot food can traumatize the mucosa. As the mucosa progressively becomes thin and friable during radiation and chemotherapy, even speaking, chewing, and swallowing can cause trauma. This results in loss of barrier function, infection, and inflammation. ${ }^{24}$

\section{Management}

\section{Oral Hygiene Maintenance}

Maintaining a proper oral hygiene protocol helps to reduce the microbial flora and maintenance of optimal $\mathrm{pH}$. This prevents secondary infection of the ulcers, thereby decreasing the chance of systemic infection. Regular brushing with a soft brush and nonabrasive toothpaste is advised. Fluoride toothpaste is advised for patients undergoing radiotherapy. ${ }^{20}$ Flossing is not advised if the gingiva is inflamed and tends to bleed. Platelet count should be monitored in such situations. In patients with thrombocytopenia, cotton swab and sponges can be used to clean the teeth instead of toothbrush to avoid trauma to the mucosa.

Bland rinses like normal saline, half a teaspoon of sodium bicarbonate in $240 \mathrm{~mL}$ of water, half a teaspoon of sodium chloride in $240 \mathrm{~mL}$ of water are suggested for frequent gargling to remove food debris, to lubricate and moisturize the oral cavity. Sugarfree gums and artificial saliva substitute can be used to combat hyposalivation. Commercial mouthwashes are not recommended as they contain alcohol, preservatives, flavoring agents, which can irritate the already inflamed mucosa. ${ }^{20}$

\section{Oral Prophylaxis}

It is the role of the dentist to treat any preexisting disease of the oral cavity. Root stumps, mobile teeth, impacted, and grossly decayed teeth should be extracted 2 weeks prior to the commencement of cancer therapy. The goal is to minimize any invasive procedure during and immediately after the cancer therapy. Extractions should be done with minimum trauma to the hard and soft tissues.

Periodontitis is an inflammatory disease that results in progressive loss of the tooth-supporting structures. Active infections should be treated aggressively with anti-inflammatory and antimicrobial drugs. After the periodontal therapy, the patient should be educated for lifelong regular follow-up.

Existing frank and incipient carious lesions should be restored. Irradiated oral environment is conducive for the development of a rapid and rampant form of caries called radiation caries. Prevention of radiation caries is important as the oral environment becomes altered and hostile for restoration postradiation. Radiation affects the vascularity of teeth within the field of radiation, resulting in micromorphological changes in the dentin, DEJ, and enamel. ${ }^{25}$ This could pose a problem if adhesive restorative materials are to be used. Prevention may not be possible always. If radiation caries is diagnosed, it should be restored swiftly. Studies have confirmed that glass ionomer cement has a better adhesive property and prevents secondary caries due to its fluoride releasing property in radiation caries. ${ }^{26,27}$ Patients are counseled to use topical fluoride and remineralization paste regularly. Coronoplasty is done for teeth with sharp margins to avoid mucosal irritation.

\section{Denture Care}

Mucosal trauma contributes to the severity of mucositis. III-fitting dentures should be altered or lined with tissue conditioners to reduce friction. Patients should be advised to maintain proper denture hygiene and advised against prolonged denture wearing. If ulcers are present, dentures are to be worn only during mealtime 
or avoided until the ulcers heal. ${ }^{20}$ Making new prosthesis should be deferred until healing is complete.

\section{Diet}

Adequate hydration should be emphasized with a well-balanced diet. The food should be soft, moist, and bland. Patients should avoid spicy, hot, dry, and coarse food. High carbohydrate and sugary food should be avoided. Patients should be advised against using caffeine, alcohol, and tobacco. ${ }^{20}$

\section{Antimicrobial Therapy}

Immune compromised patients with oral mucositis are prone to develop secondary bacterial, fungal, and viral infections. The cause of infection is identified and treated with specific topical or systemic antimicrobial therapy. Topical therapy may not be effective due to the need for multiple applications and patient compliance factors. While choosing systemic therapy over topical drug therapy drug interaction, and side effects should be taken into consideration. ${ }^{28}$

\section{MASCC/ISOO Guidelines}

The Multinational Association of Supportive Care in Cancer and International Society of Oral Oncology (MASCC/ISOO) provide guidelines and recommendations for prophylactic, therapeutic, and palliative care of patients with oral mucositis. It provides evidencebased clinical practice guidelines for mucositis. The panel provides "recommendation" only if there are more than one well-designed randomized control trials without any major flaws. It provides "suggestions" if the evidence is provided by nonrandomized and nonexperimental studies. It provides a guideline "against" the use of an agent if there were adequate evidence showing the lack of efficiency. ${ }^{29}$

\section{MASCC/ISOO Recommendations and Suggestions in Favor of Intervention}

\section{Cryotherapy}

Cryotherapy is recommended for the prevention of chemotherapyinduced oral mucositis. It is used only for short bolus doses of 5-fluorouracil, melphalan, and edatrexate. It involves holding ice chips or popsicles in the mouth 5 minutes, prior to the infusion of the drug during infusion, and 30 minutes after. ${ }^{28}$ Localized vasoconstriction and reduced blood flow to the region reduces the amount of drug delivered to the oral cavity and hence reduces drug toxicity. It is well-tolerated, albeit with some degree of discomfort.

\section{Low-level Laser Therapy}

It involves using low levels of infrared and near-infrared rays from coherent (LASER) or noncoherent (LED) light sources on the tissues to elicit various tissue responses, like enhancement of tissue repair, prevention and reduction of inflammation, tissue damage, and pain. It is also referred to as photobiostimulation as the photons from the light sources are absorbed by the mitochondrial chromophores leading to an alteration in the cellular activity. ${ }^{30}$ Multinational Association of Supportive Care in Cancer and International Society of Oral Oncology clinical practice guidelines suggest the use of low-level laser (light) therapy (LLLT) $(632.5-650 \mathrm{~nm}$ ) for the prevention of oral mucositis in patients undergoing radiotherapy and chemotherapy. ${ }^{29}$

\section{Pain Management}

A varying degree of pain from a mild burning sensation to severe pain may be experienced by the patients. Severe pain interferes with oral hygiene measures and food intake, leading to increased risk of local and systemic infection, malnutrition, and dehydration. Patientcontrolled analgesia with morphine is recommended for treating oral mucositis-related pain in patients undergoing hematopoietic stem cell transplant. In patients undergoing a moderate dose of radiotherapy (50 Gy) for head and neck cancer without concomitant chemotherapy, the panel recommends benzydamine mouthwash to prevent oral mucositis. It is a locally acting analgesic and antiinflammatory agent. The panel suggests the use of transdermal fentanyl, $2 \%$ morphine mouthwash and $0.5 \%$ doxepin mouthwash for controlling pain due to oral mucositis. ${ }^{29}$

\section{Zinc Supplement}

Zinc, a micronutrient is a cofactor for many enzymes maintaining cellular homeostasis, immune response, and wound healing. It is an antioxidant that prevents oxidative damage to many biomolecules. ${ }^{31}$ Multinational Association of Supportive Care in Cancer and International Society of Oral Oncology clinical practice guidelines suggest the use of oral zinc supplements to prevent oral mucositis in patients undergoing radiation and chemotherapy. ${ }^{29}$

\section{Keratinocyte Growth Factor}

Keratinocyte growth factor is produced by endothelial and T cells in the submucosa. It triggers the basal cells and keratinocytes in the overlying epithelium to migrate, proliferate, and differentiate. It also reduces epithelial apoptosis and attenuates the ROS that mediate epithelial injury. ${ }^{14}$ Recombinant human KGF (KGF-1/palifermin) is recommended for preventing oral mucositis in patients undergoing myelosuppressive chemotherapy and total body radiation prior to autologous stem cell transplant. It is given at a dose of $60 \mu \mathrm{g} / \mathrm{kg}$ per day for 3 days prior to conditioning treatment and 3 days after transplant. KGF-1 increases the thickness of the epithelium, decreases the severity and pain. ${ }^{29}$ It reduces the need of opioid drug for pain management. However, the cost of the drug could be a major concern for patients. ${ }^{32}$

\section{Recommendation and Suggestion against an Intervention}

Sucralfate forms a barrier on the mucosa and protects against acid and enzyme attack. It is prescribed to facilitate the healing of gastric and duodenal ulcers. Sucralfate mouthwash is not recommended to prevent or treat oral mucositis in patients receiving radiotherapy and chemotherapy. ${ }^{29}$ This is because the primary damage occurs first in the submucosa, and the loss of epithelium occurs later as a secondary effect. ${ }^{14}$

Chlorhexidine mouthwash is prescribed for treating gingivitis and preventing plaque formation. It exhibits antifungal and antibacterial activity by disrupting the microbial cell membrane and coagulating the intracellular molecules. ${ }^{33}$ Chlorhexidine mouthwash is not recommended for the prevention of oral mucositis in patients undergoing head and neck radiotherapy. ${ }^{29}$ This can be attributed to the fact that infection is not a primary event in the etiopathogenesis of oral mucositis. ${ }^{13}$ Also, long-term use of chlorhexidine can lead to staining of teeth and dorsum of the tongue, formation of calculus, and infrequently mucosal desquamation and soreness. ${ }^{33}$

\section{Discussion}

Although multiple oral factors like poor oral hygiene, hyposalivation, mucosal trauma contribute to the severity and progress of oral mucositis, periodontitis can be seen as a prime factor. Studies have confirmed that active periodontal disease can potentiate oral 
mucositis and is also a lifelong risk factor for osteoradionecrosis postradiation. ${ }^{34}$ Head and neck radiotherapy lead to rapid loss of tooth-supporting structures. ${ }^{35}$

While mild subepithelial inflammatory cell infiltrate can stimulate mitosis, severe inflammation hampers the proliferation of the cells. ${ }^{6}$ Controlling inflammation and infection in the oral cavity thus gains significance and is the treatment goal for both periodontitis and oral mucositis. Sub antimicrobial dose of doxycycline has been proved to be effective in controlling the inflammatory response in periodontal disease. Sub antimicrobial dose of doxycycline downregulates the enzymes MMPs responsible for the destruction of extracellular matrix in the connective tissue. ${ }^{36}$ A randomized clinical trial done to evaluate the effect of sub antimicrobial dose of doxycycline on chemotherapy-induced oral mucositis proved to be ineffective. ${ }^{37}$ More research is needed to ascertain the efficacy of sub antimicrobial dose of doxycycline in treating oral mucositis. Developing comprehensive treatment plans to control the infection and inflammation in the oral cavity is essential. Since, these effects might influence changes in oral physiology, which in turn can modify the therapeutic outcome of radiotherapy by altering the immune response of the cells.

\section{Conclusion}

Management of oral mucositis is an interdisciplinary approach involving oncologists, physicians, dentists, and dieticians. However, it is the dentist who plays a crucial role in controlling the course of the condition before, during, and after the completion of cancer therapy. Hence, communication between the oncologist and the dentist becomes vital. Advanced cancer, immunocompromised state, and severe malnutrition might preclude the patient from undergoing a standard dental treatment protocol before the commencement of cancer therapy. The dental surgeon should record the baseline oral status before the commencement of cancer therapy so that any deviation can be observed immediately.

The dentist should be made aware of the dosage, duration of chemo and radiotherapy; the field of radiation; specification of the chemotherapeutic drug. The degree of mucositis to be anticipated, along with the proposed treatment protocol can then be conveyed to the patient. Since depression is a coexisting condition in most patients undergoing cancer therapy, patients may not feel motivated to maintain oral hygiene. The patient can also become debilitated during the course of the treatment. To handle such situations, the attending family members along should also be counseled, explaining the significance of maintaining oral hygiene. ${ }^{38}$ The oral hygiene protocol advised should be simple and practical for the patient and the family members. The therapeutic and preventive drugs prescribed should also be easily available and cost-effective for sustainable oral care.

\section{Authors' Contributions}

The work was conceptualized by Sathyasree Madeswaran, Kavitha Muthu, and Saravanan R. Sathyasree Madeswaran, Kavitha Muthu, and Deepshika Saravanan did the methodology. Sathyasree Madeswaran and Saravanan R did the formal analysis and writing. Saravanan R, Kavitha Muthu, and Deepshika Saravanan did the review and editing.

\section{References}

1. Sroussi HY, Epstein JB, Bensadoun RJ, et al. Common oral complications of head and neck cancer radiation therapy: mucositis, infections, saliva change, fibrosis, sensory dysfunctions, dental caries, periodontal disease, and osteoradionecrosis. Cancer Med 2017;6(12):2918-2931. DOI: 10.1002/cam4.1221.

2. White $S$, Pharoah M. Oral radiology: principles and interpretation, 7th ed. St. Louis: Elsevier; 2014.

3. Vera-Llonch M, Oster G, Ford CM, et al. Oral mucositis and outcomes of allogenic hematopoietic stem-cell transplantation in patients with hematologic malignancies. Support Care Cancer 2007;15(5):491-496. DOI: 10.1007/s00520-006-0176-9.

4. Vera-Llonch $M$, Oster $G$, Hagiwara M, et al. Oral mucositis in patients undergoing radiation treatment for head and neck carcinoma. Cancer 2006;106(2):329-336. DOI: 10.1002/cncr.21622.

5. Forman SJ, Negrin RS, Antin JH, et al. Thomas' Hematopoietic Cell Transplantation. Hoboken, United Kingdom: John Wiley \& Sons, Incorporated; 2015.

6. Squier C, Brogden K. Human oral mucosa. Chichester, West Sussex, U.K.: Wiley-Blackwell; 2011.

7. Sonis S. A biological approach to mucositis. J Support Oncol 2003;2(1):21-32.

8. Vissink A, Jansma J, Spijkervet FKL, et al. Oral Sequelae of Head and Neck Radiotherapy. Crit Rev Oral Biol Med 2003;14(3):199-212. DOI: 10.1177/154411130301400305.

9. Chaveli-López B, Bagán-Sebastián JV. Treatment of oral mucositis due to chemotherapy. J Clin Exp Dent 2016;8(2):201-209. DOI: 10.4317/ jced.52917.

10. Elting LS, Cooksley CD, Chambers MS, et al. Risk, outcomes, and costs of radiation-induced oral mucositis among patients with head-andneck malignancies. Int J Radiat Oncol Biol Phys 2007;68(4):1110-1120. DOI: 10.1016/j.ijrobp.2007.01.053.

11. Elting LS, Coakley C, Chambers M, et al. The burdens of cancer therapy: clinical and economic outcomes of chemotherapy-induced mucositis. Cancer 2003;98(7):1531-1539. DOI: 10.1002/cncr.11671.

12. Bloomer WD, Hellman S. Normal tissue responses to radiation therapy. N Engl J Med 1975;293(2):80-83. DOI: 10.1056/ NEJM197507102930206.

13. Donnelly JP, Bellm LA, Epstein JB, et al. Antimicrobial therapy to prevent or treat oral mucositis. Lancet Infect Dis 2003;3(7):405-412. DOI: 10.1016/S1473-3099(03)00668-6.

14. Sonis S. Pathobiology of oral mucositis: novel insights and opportunities. J Support Oncol 2007;5(9 Suppl 4):3-11.

15. Jensen S, Pedersen A, Reibel J, et al. Xerostomia and hypofunction of the salivary glands in cancer therapy. Support Care Cancer 2003;11(4):207-225. DOI: 10.1007/s00520-002-0407-7.

16. Jose-Louis P, Andres A, Philippe N. Mucositis: its occurrence, consequences, and treatment in the oncology setting. Oncologist 1998;3(6):446-451.

17. Lustberg M. Management of neutropenia in cancer patients. Clin Adv Hematol Oncol 2012;10(12):825-826.

18. Van Der Velden W, Herbers A, Netea M, et al. Mucosal barrier injury, fever and infection in neutropenic patients with cancer: introducing the paradigm febrile mucositis. Br J Haematol 2014;167(4):441-452. DOI: 10.1111/bjh.13113.

19. Kaufman RM, Anderson KC. Causes of Pancytopenia. Holland-Frei Cancer Medicine, 6th ed. Hamilton (ON): BC Decker; 2003.

20. BC Cancer Agency. Symptom Management Guidelines: Oral mucositis. [Internet]. 2019 [cited 27 April 2019]. Available from: http://www. bccancer.bc.ca/nursing site/Documents/12.\%200ral\%20Mucositis. pdf.

21. Argilés JM. Cancer-associated malnutrition. Eur J Oncol Nurs 2005;9(Suppl 2):S39-S50. DOI: 10.1016/j.ejon.2005.09.006.

22. Ziętarska M, Krawczyk-Lipiec J, Kraj L, et al. Chemotherapy-related toxicity, nutritional status and quality of life in precachectic oncologic patients with, or without, high protein nutritional support. A prospective, randomized study. Nutrients 2017;9(10):E1108. DOI: 10.3390/nu9101108.

23. Squier CA, Kremer MJ. Biology of oral mucosa and esophagus. J Natl Cancer Inst Monogr 2001;29:7-15. DOI: 10.1093/oxfordjournals. jncimonographs.a003443. 
24. Treister NS, Chemotherapy-Induced Oral Mucositis [Internet]. 2017. Available from: https://emedicine.medscape.com/article/1079570overview.

25. Springer IN, Niehoff P, Warnke PH, et al. Radiation caries - radiogenic destruction of dental collagen. Oral Oncol 2005;41(7):723-728. DOI: 10.1016/j.oraloncology.2005.03.011.

26. McComb D, Erickson RL, Maxymiw WG, et al. A clinical comparison of glass ionomer, resin-modified glass ionomer and resin composite restorations in the treatment of cervical caries in xerostomic head and neck radiation patients. Oper Dent 2002;27(5):430-437.

27. Hu JY, Li YQ, Smales RJ, et al. Restoration of teeth with more-viscous glass ionomer cements following radiation-induced caries. Int Dent J 2002;52(6):445-448. DOI: 10.1111/j.1875-595X.2002.tb00640.x.

28. Peterson DE, Jensen SB. Oral complications of nonsurgical cancer therapies: diagnosis and treatment. In: Glick M, ed. Burket's Oral Medicine, 12th ed. People's Medical Publishing House; 2014. pp. 201-218.

29. Lalla R, Bowen J, Barasch A, et al. MASCC/ISOO clinical practice guidelines for the management of mucositis secondary to cancer therapy. Cancer 2014;120(10):1453-1461. DOI: 10.1002/cncr. 28592.

30. Avci P, Gupta A, Sadasivam M, et al. Low-level laser (light) therapy (LLLT) in skin: stimulating, healing, restoring. Semin Cutan Med Surg 2013;32(1):41-52.

31. Lin $P$, Sermersheim $M$, Li H, et al. Zinc in wound healing modulation. Nutrients 2017;10(1):E16. DOI: 10.3390/nu10010016.
32. Nooka A, Johnson H, Kaufman J, et al. Pharmacoeconomic analysis of palifermin to prevent mucositis among patients undergoing autologous hematopoietic stem cell transplantation. Biol Blood Marrow Transplant 2014;20(6):852-857. DOI: 10.1016/ j.bbmt.2014.02.025.

33. Yagiela J, Dowd F, Johnson B, et al. Pharmacology and therapeutics for dentistry, 6th ed. Elsevier Health Sciences; 2010. pp. 735-750.

34. Katsura K, Sasai K, Sato M, et al. Relationship between oral health status and development of osteoradionecrosis of the mandible: a retrospective longitudinal study. Oral Surg Oral Med Oral Pathol Oral Radiol Endod 2008;105(6):731-738. DOI: 10.1016/j.tripleo.2007.10.011.

35. Epstein JB, Lunn R, Le N, et al. Periodontal attachment loss in patients after head and neck radiation therapy. Oral Surg Oral Med Oral Pathol Oral Radiol Endod 1998;86(6):673-677. DOI: 10.1016/S10792104(98)90202-5.

36. Preshaw PM, Hefti AF, Jepsen $S$, et al. Subantimicrobial dose doxycycline as an adjunctive treatment for periodontitis. A review. J Clin Periodontol 2004;31(9):697-707. DOI: 10.1111/j.1600051X.2004.00558.x.

37. Ramirez-Amador V, Anaya-Saavedra G, Labardini-Mendez J, et al. Double-blind placebo-controlled randomized clinical trial evaluating doxycycline effects on chemotherapy-induced oral mucositis. J Clin Pharm Ther 2018;43(2):202-208. DOI: 10.1111/jcpt.12633.

38. Brown C, Yoder L. Stomatitis: an overview. In: The American Journal of Nursing, Supplement: Oncology Nursing Update. Lippincott Williams \& Wilkins; 2002. pp. 20-23. 